\title{
Morbidity and Mortality Associated With Pediatric Critical Mediastinal Mass Syndrome
}

Saad Nasir ${ }^{1}$, Rafia Jabbar ${ }^{2}$, Faiza Rehman ${ }^{2}$, Muhammad Khalid ${ }^{3}$, Muhammad Rahil Khan ${ }^{2}$, Anwar Haque $^{2}$

1. Internal Medicine, United Medical and Dental College, Creek General Hospital, Karachi, PAK 2. Pediatrics, The Indus Hospital, Karachi, PAK 3. Pediatrics, The Children's Hospital \& The Institute of Child Health, Multan, PAK

Corresponding author: Saad Nasir, saad-nasir@hotmail.com

\section{Abstract \\ Objective}

The critical mediastinal mass syndrome (CMMS) is a life-threatening condition and is challenging for physicians. We analyse the clinicopathological profile and outcome of CMMS from a large tertiary-care pediatric oncology center in Pakistan.

\section{Methods}

We retrospectively reviewed the medical record of a tertiary-care hospital in Pakistan from April 2017 to September 2019 for all children (1 month-16 years) who presented with an anterior mediastinal mass (AMM). A CMMS case is defined as a child with an AMM presenting with cardiorespiratory compromise and needing intensive care support. Demographic data, clinical profile, pathological diagnosis, and outcome of all such children were recorded. Descriptive statistics were applied using the Statistical Package for the Social Sciences (SPSS), version 22 (IBM Corp., Armonk, NY).

\section{Results}

Of the total 221 mediastinal masses, 61 children were diagnosed as CMMS and enrolled in the study. The mean age was $9 \pm 3.3$ years, and $68.9 \% \%$ were male; $65.6 \%$ of patients had a weight for age less than the fifth percentile. A total of $49.2 \%$ of patients had a duration of illness of more than one month before diagnosis. Fever (97.6\%) and lymphadenopathy (82\%) were the most common findings, along with respiratory and cardiovascular signs and symptoms; $9.8 \%$ had superior vena cava syndrome. The pericardial effusion was present in $54.6 \%$ and $27.9 \%$ had pleural effusion. Peripheral blood flow cytometry made the diagnosis in $59 \%$, peripheral lymph node biopsy in $13 \%$, mediastinal core biopsy in $5 \%$, and pleural fluid flow cytometry in one case; $62.3 \%$ had a white blood cell count of $>100,000 / \mathrm{mm}^{3}$. A total of $72.1 \%(\mathrm{n}=44)$ cases were diagnosed as T-cell acute lymphoblastic leukemia in our cohort. Clinical and laboratory tumor lysis syndrome developed in $10 \%$ and $73 \%$ of cases, respectively. Mechanical ventilation was required in $9.8 \%$ of the cohort. Mortality was reported in 10 (16.4\%) patients.

Received 06/07/2020

Review began 06/15/2020 Review ended 06/16/2020 Published 06/26/2020

\section{(c) Copyright 2020}

Nasir et al. This is an open access article distributed under the terms of the Creative Commons Attribution License CC-BY 4.0., which permits unrestricted use, distribution, and reproduction in any medium, provided the original author and source are credited.

\section{Conclusion}

We found that the $100 \%$ fatality rate with controlled positive pressure ventilation and spontaneous breathing is ideal. Tumour lysis syndrome was the most common morbidity in our cohort.

\author{
Categories: Pediatrics, Oncology \\ Keywords: pediatric cancers, anterior mediastinal mass, critical, acute lymphoblastic leukemia
}

\section{Introduction}

Anterior mediastinal mass (AMM) is common in pediatric age, but the incidence is unknown [1]. AMM in children has varied presentations from asymptomatic to life-threatening events like respiratory arrest or cardiovascular collapse and even death. The most common presentations of childhood AMM are similar to symptoms and signs seen in common respiratory illnesses [2]. Several clinical reports described lifethreatening events in AMM in children during treatment, especially related to procedures or postural changes either as acute respiratory or cardiovascular compromise [3,4]. Children are more vulnerable due to a small thoracic cavity size, more compressible cartilaginous structure of the airway, and higher oxygen consumption rate with little reserve [5]. AMM in children is challenging, and sometimes a nightmare situation for pediatric oncologists and pediatric intensivists. A study described one spectrum of childhood AMM presenting with respiratory symptoms and signs or symptoms and signs of superior vena cava syndrome, presence of pleural effusion and/or pericardial effusion or radiological evidences of compression/deviation of the airway or great vessel as critical mediastinal mass syndrome (CMMS) [6]. These cases need a multidisciplinary team approach for diagnostic, therapeutic, and supportive care to improve the outcome and avoid preventable complications to decrease morbidity and mortality $[7,8]$. 
Published research on CMMS is limited; hence, we investigated these cases in the present study to determine the mortality and morbidity in CMMS. Our secondary aim was to determine the clinicopathological profile of these patients.

\section{Materials And Methods}

A retrospective cohort study was done from April 2017 to September 2019 at the Pediatric Intensive Care Unit (PICU) of a tertiary-care center with a very high burden of pediatric oncology patients, registering more than 800 new cancers in children annually. The study participants included all children (age range: 1 month16 years) with anterior mediastinal mass presenting acutely with cardiorespiratory symptoms being admitted in PICU. Age, gender, ethnicity, weight, nutritional status, duration of illness along with clinical variables and outcome variables (morbidity [defined complications occurred in PICU], and mortality as death in the same admission) were collected on a structured data collection sheet. Patients confidentiality was maintained. This study aimed to determine the clinicopathological profile and outcomes of CMMS. Ethical approval to conduct the study was obtained from the Institutional Review Board of the Indus Hospital (IRD_IRB_2019_09_012).

CMMS was defined as a mass in the anterior mediastinum that causes respiratory signs and symptoms, signs of superior vena cava syndrome, radiological evidence of compression, deviation of the airway, or great vessel or presence of pleural/pericardial effusion [6]. Hyperleukocytosis was defined as a peripheral white blood cell count greater than 100,000/mL. Morbidity was defined as a respiratory failure (use of mechanical ventilation), sepsis (empirical use of broad-spectrum antibiotics [vancomycin, meropenem, and colistin] with clinical suspicion of sepsis), shock (use of any inotrope or vasopressor), acute kidney injury (rise in serum creatinine based on Kidney Disease: Improving Global Outcomes [KDIGO] criteria) and tumor lysis syndrome (laboratory or clinical based on Bishop's criteria) $[9,10]$.

Data were entered into and analysed using the Statistical Package for the Social Sciences (SPSS), version 22 (IBM Corp., Armonk, NY). Means and standard deviations were calculated for continuous variables such as age, laboratory characteristics, and length of hospital stay. Frequency and proportions were used for categorical variables such as gender, and clinical and laboratory characteristics. Simple descriptive statistics were applied. Univariate logistic regression analysis was performed to assess the ability of variables such as age, gender, nutritional status, duration of symptoms, diagnosis, and laboratory findings including, total leukocyte count and platelet count, to predict the mortality outcome.

\section{Results}

Of the total 221 mediastinal mass cases, 61 (27.6\%) children were enrolled during the study period. The mean age was $9 \pm 3.34$ years, and there was a male predominance $(68.9 \%) ; 65.6 \%(n=40)$ of patients had a weight for age less than the fifth percentile, while the mean weight was $23.2 \pm 10 \mathrm{~kg}$. Approximately half of the patients had the illness for one month before getting diagnosed with this condition. Other demographic features are mentioned in Table 1. 


\section{Cureus}

Variable

n (\%)

Age groups

2-5 years

11 (18.0)

6-10 years

33 (54.1)

$>10$ years

17 (27.9)

Gender

Male

42 (68.9)

Female

Ethnicity

Sindhi

Punjabi

31 (50.8)

01 (1.6)

Balochi

18 (29.5)

Not known

11 (18.0)

Weight $(\mathrm{kg})$ mean $\pm \mathrm{SD}$

$23.2 \pm 10$

Malnourished

Yes

$40(65.6)$

No

21 (34.4)

Duration of illness

$<1$ week

1-2 weeks

09 (14.8)

>2-3 weeks

03 (4.9)

>3-4 weeks

$11(18.0)$

$>4$ weeks

30 (49.2)

Length of stay in days (mean $\pm \mathrm{SD}$ )
$6 \pm 5.23$ (range 1-32)

TABLE 1: Demographic characteristics of children presenting with critical mediastinal mass $(n=61)$

n, sample size.

Most of these children presented with nonspecific symptoms such as fatigue (63.9\%), followed by weight loss, respiratory distress, cough, while fever (96.7\%) and lymphadenopathy (82\%) were the most frequent clinical signs present (Table 2). Six (9.8\%) patients had superior vena cava syndrome. 


\section{Cureus}

Clinical characteristic

n (\%)

Presenting symptoms

Cough

$16(26.2)$

Respiratory distress

$18(29.5)$

Weight loss

23 (37.7)

Fatigue

39 (63.9)

Headache

$13(21.3)$

Others $^{a}$

07 (11.5)

Clinical signs

Fever

59 (96.7)

Tachypnea

44 (72.1)

Decrease air entry

Crepitations in lungs

15 (24.6)

Desaturation

24 (39.3)

Lymphadenopathy

50 (82.0)

Hepatosplenomegaly

46 (75.4)

SVCS

$06(9.8)$

Other clinical signs ${ }^{\mathrm{b}}$

19 (31.2)

TABLE 2: Clinical characteristics of children presenting with critical mediastinal mass $(n=61)$

aOther symptoms include chest pain, dysphagia, night sweats, and wheeze.

bOther clinical signs include facial swelling, upper body edema, stridor, ronchi, and decreased level of consciousness.

n, sample size; SVCS, superior vena cava syndrome.

Our data showed the presence of hyperleukocytosis in 38 (62.3\%) patients, while more than half of the children had positive findings on chest X-ray, including pleural effusion in 17 (27.9\%) patients. Echocardiography done in 55 patients showed pericardial effusion in $54.6 \%$ of patients, while three cases had partial great vessel obstruction (Table 3). 


\section{Cureus}

Laboratory characteristics

n (\%)

Hematology profile

Hemoglobin $(g / d L),($ mean $\pm S D)$

$7.38 \pm 2.70$

Platelet counts

$<50,000\left(\right.$ per $\left.\mathrm{mm}^{3}\right)$

$41(67.2)$

$50,000-100,000\left(\right.$ per $\left.\mathrm{mm}^{3}\right)$

$06(9.8)$

$>100,000\left(\right.$ per mm $\left.{ }^{3}\right)$

$14(23.0)$

Leucocyte counts

$<10,000 / \mathrm{mL}$

05 (8.2)

$10,000-50,000 / \mathrm{mL}$

$16(26.2)$

$51,000-100,000 / \mathrm{mL}$

02 (3.3)

$>100,000 / \mathrm{mL}$

$38(62.3)$

Deranged coagulation profile

$10(16.4)$

Positive smear for blast cells

$49(80.3)$

Serum potassium $(\mathrm{mmol} / \mathrm{L})($ mean $\pm \mathrm{SD})$

$3.97 \pm 1.03$

Serum uric acid $(\mathrm{mg} / \mathrm{dL})($ mean $\pm \mathrm{SD})$

$7.90 \pm 4.10$

Serum LDH (mini-max)

$361-5646$

Serum creatinine $(\mathrm{mg} / \mathrm{dL})($ mean $\pm \mathrm{SD})$

$0.5 \pm 0.24$

Serum phosphate $(\mathrm{mg} / \mathrm{dL})($ mean $\pm \mathrm{SD})$

$3.75 \pm 0.91$

Alanine aminotransferase (mean $\pm \mathrm{SD}$ )

$42.85 \pm 54.39$

Positive blood culture

07 (11.5)

Radiological profile

Chest X-ray performed

$61(100)$

Effusion on chest X-ray

17 (27.9)

Lung abnormalities

$19(31.1)$

CT-scan chest performed

Effusion on CT chest

03 (37.5)

Tracheal compression

01 (1.6)

Vessel compression

02 (3.2)

Echocardiography done

$55(90.2$

Pericardial effusion

$30(54.6)$

Vessel compression

03 (5.5)

Ejection fraction (min-max)

50-87

TABLE 3: Laboratory characteristics of children presenting with critical mediastinal mass $(n=61)$

$\mathrm{n}$, sample size; LDH, lactate dehydrogenase.

Figure 1 shows that the most common diagnosis was T-cell acute lymphoblastic leukemia (72.1\%), while $4.4 \%$ had lymphoblastic lymphoma. Peripheral blood flow cytometry was used to make the diagnosis in most 


\section{Cureus}

(59\%) of these children, followed by peripheral lymph node biopsy (13.1\%). A total of 22 (32.6\%) patients developed tumor lysis syndrome as a complication. The mean length of hospital stay was $6 \pm 5.2$ days. Analysis of the outcomes revealed that mortality was observed in 10 (16.4\%) patients, while all six patients in our cohort, who were put on mechanical ventilation, did not survive (Table 4).

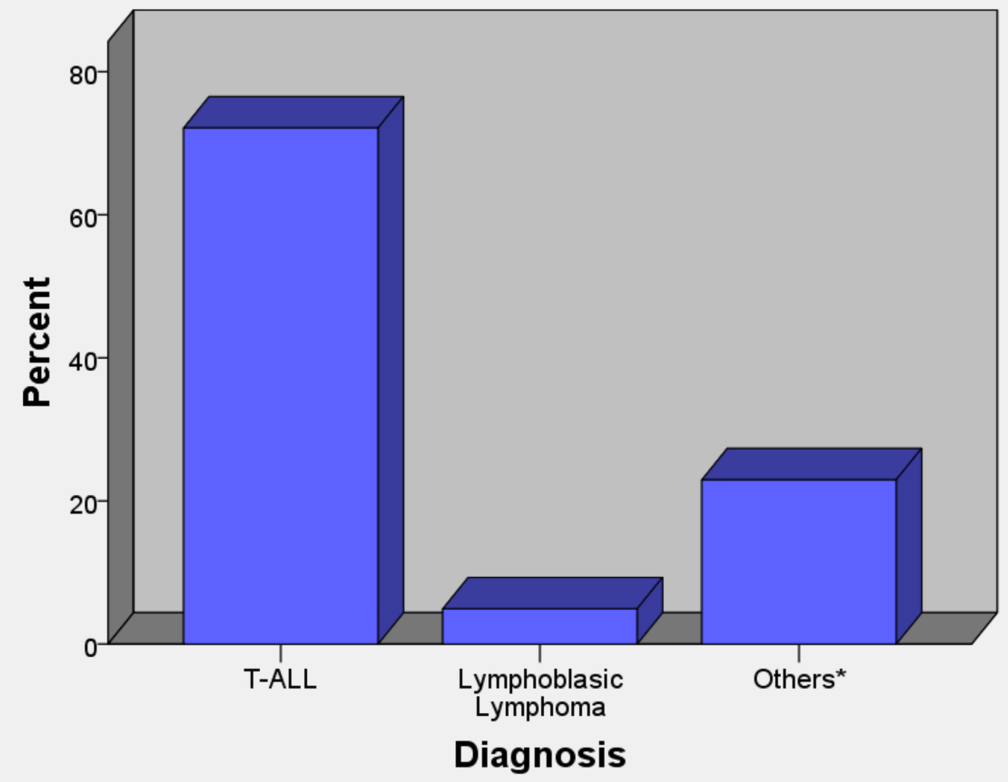

FIGURE 1: Diagnosis in patients with anterior mediastinal masses $(n=61)$

*Neuroblastoma, Ewing sarcoma, B-cell non-Hodgkin's lymphoma, suspected leukemia died before diagnosis, and suspected Hodgkin's lymphoma.

T-ALL, T-cell acute lymphoblastic leukemia. 


\section{Cureus}

Characteristic

n (\%)

Mode of diagnosis

On peripheral blood flow cytometry

On pleural fluid flow cytometry

01 (1.6)

On mediastinal mass core biopsy

03 (4.9)

On peripheral lymph node biopsy

08 (13.1)

Complications

Septic shock

$13(21.3)$

Acute kidney injury on admission

05 (8.2)

Acute kidney injury during treatment

07 (11.5)

TLS

$22(36.1)$

Clinical TLS

02 (9.1)

Laboratory TLS

$16(72.7)$

Both clinical and laboratory TLS

05 (22.7)

Intervention

Cytoreduction

60 (98.4)

Chemotherapy

Both chemotherapy and LP

Pre-treatment steroids

$04(6.6)$

Inotropic support

08 (13.1)

Mechanical ventilation

$06(9.8)$

Dialysis

Cardiopulmonary resuscitation

$04(6.6)$

Outcome

Mortality

10 (16.4)

TABLE 4: Mode of diagnosis, intervention, and outcome of children presenting with critical mediastinal mass $(n=61)$

n, sample size; TLS, tumor lysis syndrome; LP, lumbar puncture.

A univariate logistic regression analysis was calculated to predict participants' outcomes based upon other variables such as age, gender, nutritional status, duration of symptom, diagnosis, total leukocyte count, platelet count, and mechanical ventilation. We found a non-significant relationship between all the other predictor values ( $p$-value $>0.05$ ), except mechanical ventilation and the mortality outcome (p-value $<0.001$ ) (Table 5). 


\section{Cureus}

\begin{tabular}{|c|c|c|c|}
\hline Determinant & Odds ratio & $95 \% \mathrm{Cl}$ & p-value \\
\hline Age (yrs) & 0.81 & $0.65-1.00$ & 0.05 \\
\hline \multicolumn{4}{|l|}{ Gender } \\
\hline Male & 1 & - & - \\
\hline Female & 1.07 & $0.24-4.67$ & 0.93 \\
\hline \multicolumn{4}{|l|}{ Malnourished } \\
\hline No & 1 & - & - \\
\hline Yes & 0.79 & $0.18-3.41$ & 0.75 \\
\hline \multicolumn{4}{|l|}{ Duration of symptoms } \\
\hline Up to 2 weeks & 1 & - & - \\
\hline >2-4 weeks & 0.54 & $0.09-2.94$ & 0.47 \\
\hline >4 weeks & 1.93 & $0.34-10.83$ & 0.46 \\
\hline \multicolumn{4}{|l|}{ Diagnosis } \\
\hline Others & 1 & - & - \\
\hline T-ALL & 3.25 & $0.80-13.16$ & 0.09 \\
\hline \multicolumn{4}{|l|}{ Total leucocyte count } \\
\hline$<100,000 / \mathrm{mL}$ & 1 & - & - \\
\hline$\geq 100,000 / \mathrm{mL}$ & 0.79 & 0.18-3.42 & 0.75 \\
\hline \multicolumn{4}{|l|}{ Platelet count } \\
\hline$>100,000\left(\right.$ per mm $\left.{ }^{3}\right)$ & 1 & - & - \\
\hline$<100,000\left(\right.$ per mm $\left.{ }^{3}\right)$ & 0.81 & $0.15-4.36$ & 0.81 \\
\hline \multicolumn{4}{|l|}{ Mechanical ventilation } \\
\hline Yes & 2.5 & $1.17-5.34$ & $<0.001$ \\
\hline No & 1 & - & - \\
\hline
\end{tabular}

TABLE 5: Univariate logistic regression analysis for determinants of mortality in children presenting with critical mediastinal mass $(n=61)$

n, sample size; T-ALL, T-cell acute lymphoblastic leukemia.

\section{Discussion}

This is the first comprehensive report on children with CMMS from a PICU of a lower middle-income country. We found that the mortality rate was $16.4 \%(n=10)$ in children with CMMS. The compressive effects of these masses on the airways or the great vessels owing to small intra-thoracic volume in young children can lead to cardiorespiratory compromise, resulting in death. Various studies report $25 \%-45 \%$ mortality in these patients $[11,12]$.

T-cell acute lymphoblastic leukemia was the most common malignancy $(72 \%, \mathrm{n}=44)$ in our cohort of CMMS. Kashif et al. found T-cell acute lymphoblastic leukemia in 35\% of children with a malignant mediastinal mass and found it to be the strongest risk factor for mortality $(p<0.001)$ [11]. The reported mortality was $45 \%$ in their report. In another report from India, Mathan and Ananthamurthy described 53.3\% of T-cell acute lymphoblastic leukemia in their children with mediastinal mass [13]. Similarly, Ravindranath et al. observed very high mortality in acute lymphoblastic leukemia with a mediastinal mass [14]. However, the prognostic significance of mediastinal mass in T-cell acute lymphoblastic leukemia is controversial. We did not find any association of mortality with primary diagnosis in any study. 
The most striking clinical observation of this report is that the controlled positive pressure ventilation with muscle paralysis in these patients was associated with $100 \%$ mortality $(p<0.001)$. The positive pressure ventilation with neuromuscular blockade is detrimental, further aggravating airway obstruction and leading to cardiovascular collapse from fatal airway obstruction, which is not amenable to routine cardiopulmonary resuscitation in the absence of rigid bronchoscopy and extracorporeal life-support system. There are multiples reasons for fatal airway obstruction in children with CMMS, including small lung volume, loss of spontaneous diaphragmatic activity, decrease in chest wall muscle tone, the position of the patient, and compression effect of the tumor on airway distal to the endotracheal tube. Previously published reports demonstrated that spontaneous ventilation is ideal in such patients [15-17].

The most important dilemma of determining the primary diagnosis in such life-threatening cases is challenging and needs a multidisciplinary team approach like anesthesia, interventional radiologist, and pediatric thoracic surgeon. In most cases, the alternative tissue was used for primary diagnosis and rarely needed mediastinal tissue biopsy as in many clinical reports on symptomatic mediastinal mass in children. In our study, we established the primary diagnosis from alternative tissue: 59\% from peripheral blood flow cytometry and $13 \%$ from cervical lymph node biopsy. Hence, mediastinal tissue biopsy was needed in only $5 \%$ of cases. Garey et al. in their study diagnosed all patients with T-cell acute lymphoblastic leukemia on either peripheral blood smear (50\%) or bone marrow aspiration (50\%) [7]. Acker et al. included 69 children with an anterior mediastinal mass and most cases (81\%) needed a biopsy tissue from outside the mediastinum with minimal sedation and spontaneous breathing [18].

Clinically, children with mediastinal masses are often symptomatic of both respiratory and constitutional symptoms because of malignancy. They are treated initially for common respiratory illnesses like pneumonia and asthma [12]. Lam et al. analysed clinical records of 29 children with mediastinal mass, and an anteriorly located mass was a significant factor to develop respiratory compromise $(p=0.019)$ [6]. A total of $24.1 \%$ of children were asymptomatic at the time of diagnosis. Symptomatic children presented with cough (33.3\%), dyspnea (23.8\%), and chest pain (19\%), while one patient developed wheeze [6]. Our results show that most children had non-specific symptoms such as fatigue, weight loss, and cough while fever and tachypnea were the most common clinical manifestations. These results are comparable to other studies $[6,12,19,20]$. The demographic analysis showed the mean age at diagnosis to be 9 years, higher than that seen in the existing literature $[11,21]$. Among the patients in this study, tumor lysis syndrome was the most common (36.1\%) followed by septic shock (21\%) and acute kidney injury (11\%).

This study comprehensibly reviews the PICU course of CMMS in children and further strengthens the existing literature. Study limitations included retrospective data collection from a single institution and a small sample size.

\section{Conclusions}

We found that the $100 \%$ fatality rate with controlled positive pressure ventilation and spontaneous breathing is ideal. Tumor lysis syndrome was the most common morbidity in our cohort. The rapid use of alternative tissue for primary diagnosis in most cases and the early initiation of intensive chemotherapy may improve the outcome in these children.

\section{Additional Information}

\section{Disclosures}

Human subjects: Consent was obtained by all participants in this study. Institutional Review Board, The Indus Hospital issued approval IRD_IRB_2019_09_012. Animal subjects: All authors have confirmed that this study did not involve animal subjects or tissue. Conflicts of interest: In compliance with the ICMJE uniform disclosure form, all authors declare the following: Payment/services info: All authors have declared that no financial support was received from any organization for the submitted work. Financial relationships: All authors have declared that they have no financial relationships at present or within the previous three years with any organizations that might have an interest in the submitted work. Other relationships: All authors have declared that there are no other relationships or activities that could appear to have influenced the submitted work.

\section{References}

1. Pearson JK, Tan GM: Pediatric anterior mediastinal mass: a review article. Semin Cardiothorac Vasc Anesth. 2015, 19:248-254. 10.1177/1089253215578931

2. Saraswatula A, McShane D, Tideswell D, Burke GA, Williams DM, Nicholson JC, Murray MJ: Mediastinal masses masquerading as common respiratory conditions of childhood: a case series. Eur J Pediatr. 2009, 168:1395-1399. 10.1007/s00431-009-0933-0

3. Piastra M, Ruggiero A, Caresta E, Chiaretti A, Pulitano S, Polidori G, Riccardi R: Life-threatening presentation of mediastinal neoplasms: report on 7 consecutive pediatric patients. Am J Emerg Med. 2005, 23:76-82. 10.1016/j.ajem.2003.12.025

4. Perger L, Lee EY, Shamberger RC: Management of children and adolescents with a critical airway due to compression by an anterior mediastinal mass. J Pediatr Surg. 2008, 43:1990-1997. 
10.1016/j.jpedsurg.2008.02.083

5. Kar SK, Ganguly T, Dasgupta CS, Goswami A: Cardiovascular and airway considerations in mediastinal mass during thoracic surgery. J Clin Exp Cardiolog. 2014, 5:1-8. 10.4172/2155-9880.1000354

6. Lam JC, Chui CH, Jacobsen AS, Tan AM, Joseph VT: When is a mediastinal mass critical in a child? An analysis of 29 patients. Pediatr Surg Int. 2004, 20:180-184. 10.1007/s00383-004-1142-6

7. Garey CL, Laituri CA, Valusek PA, St Peter SD, Snyder CL: Management of anterior mediastinal masses in children. Eur J Pediatr Surg. 2011, 21:310-313. 10.1055/s-0031-1279745

8. Malik R, Mullassery D, Kleine-Brueggeney M, Atra A, Gour A, Sunderland R, Okoye B: Anterior mediastinal masses - a multidisciplinary pathway for safe diagnostic procedures. J Pediatr Surg. 2019, 54:251-254. 10.1016/j.jpedsurg.2018.10.080

9. Fujii T, Uchino S, Takinami M, Bellomo R: Validation of the kidney disease improving global outcomes criteria for AKI and comparison of three criteria in hospitalized patients. Clin J Am Soc Nephrol. 2014, 9:848-854. 10.2215/CJN.09530913

10. Cairo MS, Bishop M: Tumour lysis syndrome: new therapeutic strategies and classification. Br J Haematol. 2004, 127:3-11. 10.1111/j.1365-2141.2004.05094.x

11. Kashif RU, Faizan M, Anwar S: Pediatric malignant mediastinal masses. J Coll Physicians Surg Pak. 2019, 29:258-262. 10.29271/jcpsp.2019.03.258

12. Lerman J: Anterior mediastinal masses in children. Semin Anesth Perioperat Med Pain. 2007, 26:133-140.

13. Mathan L, Ananthamurthy A: Clinicopathological attributes of T-lymphoblastic lymphoma seen in a tertiary care centre. Clin Cancer Investig J. 2018, 7:1-8. 10.4103/ccij.ccij_67_17

14. Ravindranath Y, Kaplan J, Zuelzer WW: Significance of mediastinal mass in acute lymphoblastic leukemia . Pediatrics. 1975, 55:889-893.

15. Tütüncü AC, Kendigelen P, Kaya G: Anaesthetic management of a child with a massive mediastinal mass . Turk J Anaesthesiol Reanim. 2017, 45:374-376. 10.5152/TJAR.2017.81557

16. Williams A, Singh G, George SP: Procedural sedation for a child with a mediastinal mass and superior vena caval syndrome. J Anaesthesiol Clin Pharmacol. 2015, 31:421-424. 10.4103/0970-9185.161735

17. Stricker PA, Gurnaney HG, Litman RS: Anesthetic management of children with an anterior mediastinal mass. J Clin Anesth. 2010, 22:159-163. 10.1016/j.jclinane.2009.10.004

18. Acker SN, Linton J, Tan GM, et al.: A multidisciplinary approach to the management of anterior mediastinal masses in children. J Pediatr Surg. 2015, 50:875-878. 10.1016/j.jpedsurg.2014.09.054

19. Chen $\mathrm{CH}, \mathrm{Wu} \mathrm{KH}$, Chao YH, Weng DF, Chang JS, Lin CH: Clinical manifestation of pediatric mediastinal tumors, a single center experience. Medicine (Baltimore). 2019, 98:e16732. 10.1097/MD.0000000000016732

20. Mushtaq N, Alam MM, Aslam S, Fadoo Z, Anwar ul H: Malignant mediastinal mass in children: a single institutional experience from a developing country. J Pak Med Assoc. 2014, 64:386-389.

21. Gun F, Erginel B, Unuvar A, Kebudi R, Salman T, Celik A: Mediastinal masses in children: experience with 120 cases. Pediatr Hematol Oncol. 2012, 29:141-147. 10.3109/08880018.2011.646385 